\title{
Dynamics of Relative Agreement in Multiple Social Contexts
}

\begin{abstract}
In real world scenarios, the formation of consensus is an autoorganisation process by which actors have to make a joint assessment about a target subject being it a decision making problem or the formation of a collective opinion. In social simulation, models of opinion dynamics tackle the opinion formation phenomena. These models try to make an assessment, for instance, of the ideal conditions that lead an interacting group of agents to opinion consensus, polarisation or fragmentation. In this paper, we investigate the role of social relation structure in opinion dynamics using an interaction model of relative agreement. We present an agent-based model that defines social relations as multiple concomitant social networks and apply our model to an opinion dynamics model with bounded confidence. Moreover, we discuss the influence of complex social network topologies that capture the complexity of real-world social scenarios where actors interact in multiple contexts simultaneously. The paper builds on previous work about social space design with multiple contexts and context switching, to determine the influence of such complex social structures in a process such as opinion formation.
\end{abstract}

Keywords: opinion dynamics, consensus, bounded confidence, relative agreement, social contexts, social networks, nonlinear dynamical systems.

\section{Introduction}

Understanding trend and opinion spreading or consensus formation processes within a population is fundamental to construct coherent views and explanations for real-world events or phenomena. Examples of such processes include: a group of experts having to make a joint assessment of a certain policy, the impact of a viral marketing campaign conducted using on-line social media or even, in the context of economics and politics, an issue that influences the development of opinion dynamics studies, the voting problem. This last problem was investigated in an early model proposed by Herbert Simon [21].

Formal opinion dynamics models try to provide an understanding if not an analysis of opinion formation processes. An early formulation of these models was designed to comprehend complex phenomena found empirically in groups [10]. In particular, the work on consensus building in the context of decision-making was initialised by De Grout [8] and Lehrer [15]. Empirical studies of opinion formation in large populations have methodological limitations, as such, we use simulation, 
in particular Multi-Agent Simulation (MAS), as a methodological framework to study such phenomena in a larger scale. Most opinion dynamics simulation models are based either on binary opinions $[11,3]$ or continuous opinions $[7,12$, $6,13]$. In these models, agents update their opinions either under social influence or according to their own experience.

For a detailed analysis over some opinion dynamics model analytical and simulation results, the reader can refer to [12]. For a multidisciplinary state-ofthe-art on opinion dynamics the reader can refer to [25].

In opinion dynamics models (in particular) and Agent-Based Social Simulation (ABSS) (in general), agent interactions are guided by social space abstractions. In some models, dimensionality is irrelevant. Typically, all agents can participate in interactions with all other agents, and the notion of physical space plays no role in the simulation. Axelrod, for instance, takes a different approach in his model of dissemination of culture [4] and represents agents in an abstract bi-dimensional grid which provides structure for agents to interact with each other. In Weisbuch's bounded confidence model with social networks [24], the agents are bound by different network topologies. This last model is an example where the structure in social relations not only filters the interactions the agents can engage in, but also influences the dynamics of opinion formation.

In real-world scenarios, actors engage in a multitude of social relations different in kind and quality. Most ABSS models don't explore social space designs (such as social networks) that take into account the differentiation between coexisting social worlds. Modelling multiple concomitant social relations allows for the comprehension of a variety of real-world dynamics such as, e.g., the impact of on-line social media political campaigns or what it means socially to lose a job. Furthermore, such complex social structures are the basis for the formation of social identity $[18,9]$ and play a decisive role in auto-organised processes such as consensus formation $[3,17]$ or segregation $[19,20,17]$.

This paper is aimed at extending the line of research regarding the representation of social spaces with explicit multiple concomitant social relations. This work, described in $[2,3,17]$ presents interesting insights on how different complex social relation topologies influence consensus formation dynamics. We apply the notions of multiple social contexts to a model of continuous opinion dynamics called Relative Agreement (RA) model [6]. This model is an extension of the Bounded Confidence (BC) model $[14,7,12]$.

The work in $[2,3,17]$ explores multiple contexts applied to a simple game of consensus that can be seen as a simple binary opinion dynamics models. It is found that by considering coexisting social relations, the agent population converges to a global consensus both faster and more often. This happens due to what we call permeability between contexts [2]. Permeability in multiple social contexts is created due to both social context overlapping [2] and context switching [3]. Context switching models the existence of multiple distinct social relations from which each social agent switches to and from at different instances in time. As an example, take for instance the work and family relations. 
As the RA model [6] is considerably more complex than the simple interaction game considered in $[2,3,17]$, we perform a series of experiments to determine if this social space modelling methodology exerts a similar influence in this model. This will allow to understand if the multiple-context models present properties that are transversal to the interaction processes to which they are applied.

The paper is organised as follows. In section 2 we present the opinion dynamics model along with our social space design with multiple concurrent social networks. Section 3 describes the model of experiments presenting multiple simulation scenarios and indicators for the observations. Section 4 presents the results and the corresponding discussion. Finally, in section 5 we summarise our findings and propose some future work guidelines.

\section{$2 \quad$ The proposed model}

This section describes our proposed simulation model. This new model integrates both the multi-relational modelling approach $[2,3]$ and the Relative Agreement (RA) interaction model [6]. We start by describing the multi-context model with context switching [3] and the continuous opinion dynamics model [6]. We then present the resulting ABSS model of continuous opinion formation with uncertainty, multiple social contexts and context switching dynamics.

\subsection{A model of context switching}

The multi-context approach $[2,3,17]$ considers a multitude of concomitant social relations to represent the complex social space of an agent. This setting can be seen in a simulation as a n-dimensional scenario where each dimension surface represents a different social relation (see figure 1) simulated with a social network model. Agents belong to distinct contexts (neighbourhoods) in these multiple relations.

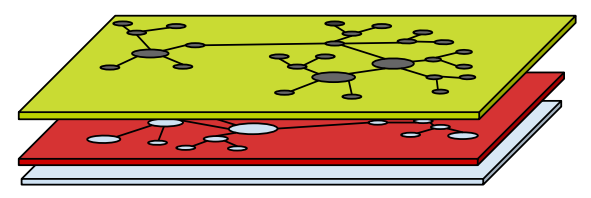

Fig. 1: Multiplex social network structure forming the social space for our models of multiple concurrent social relations.

In the particular model of context switching [3], a population of $N$ agents populates multiple social networks. Each agent is active only in one context at a time. In each simulation step, the agents select a neighbour from their current context and update their opinion according to some rule. At the end 
of each interaction an agent switches to a different context with a probability $\zeta_{c}$. For the sake of simplicity, the $\zeta_{c}$ probability is a parameter associated with each context $c$ and it is valid for all the agents in that context. This allows for modelling of time spent in each context, in an abstract way. We can think of context switching as a temporary deployment in another place, such as what happens with temporary immigration.

\subsection{Relative agreement interaction}

We now describe the model of continuous opinion dynamics with relative agreement [6]. In this model, each agent $i$ is characterised by two variables, its opinion $x_{i}$ and its uncertainty $u_{i}$ both being real numbers. The opinion values are drawn from a uniform distribution between -1 and 1 .

This model can be seen as an extension of the Bounded Confidence (BC) model $[14,7,12]$. In the $\mathrm{BC}$ model, the agents have continuous opinions and the interactions are non-linear. The agents only exert influence on each other if their opinions are within a certain fixed threshold. The threshold can be interpreted as an uncertainty, or a bounded confidence, around the opinion [6]. It is assumed that agents do not take into account opinions out of their range of uncertainty.

The RA model differs from the BC model in the fact that the change in an opinion $x_{j}$ of an agent $j$ under the influence of an agent $i$, is proportional to the overlap between the agent opinion segments (the agreement), divided by the uncertainty of the influencing agent uncertainty $u_{i}$. Another difference is that the uncertainty is not fixed, the value of $u_{j}$ is also updated using the same mechanism. The opinion and uncertainty updates are illustrated in figure 2 .

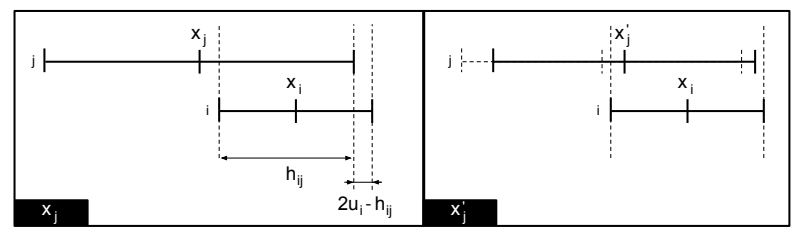

Fig. 2: Agent $i$ (with the opinion $x_{i}$ and the uncertainty $u_{i}$ ) influences agent $j$ (with the opinion $x_{j}$ and the uncertainty $u_{j}$ ). In this case, $h_{i j}$ is the overlap between the agents and $2 u_{i}-h_{i j}$ is the non-overlapping part. On the left is the representation of the opinion and uncertainty of agent $j$, on the right, the dashed lines represent the position of the segment before the interaction and the plain lines, the final values for the these two properties [6]. 
The opinion overlap $h_{i j}$ is given by:

$$
h_{i j}=\min \left(x_{i}+u_{i}, x_{j}+u_{j}\right)-\max \left(x_{i}-u_{i}, x_{j}-u_{j}\right)
$$

The opinion and uncertainty values are updated according to the following equations. As an example, the opinion $x_{j}$ and the uncertainty $u_{j}$ of agent $j$ is updated according to equation 2 and 3 respectively, if $h_{i j}>u_{i}$.

$$
\begin{aligned}
& x_{j}{ }^{\prime}=x_{j}+\mu\left(\frac{h_{i j}}{u_{i}}-1\right)\left(x_{i}-x_{j}\right) \\
& u_{j}{ }^{\prime}=u_{j}+\mu\left(\frac{h_{i j}}{u_{i}}-1\right)\left(u_{i}-u_{j}\right)
\end{aligned}
$$

Where the $\mu$ is a constant parameter which amplitude controls the speed of the dynamics. For more details, refer to [6].

\subsection{Context switching with relative agreement}

The proposed model integrates both the context switching (described in section 2.1) and the relative agreement models. In this model, agents are embedded in static social networks, interact using the opinion dynamics rules set by the RA model described in the previous section 2.2, and switch contexts (the agent neighbourhood in the network) according to a probability $\zeta_{C_{k}}$, associated with each context $C_{k}$.

Our proposed simulation model behaves as follows. Consider a population of $N$ agents distributed by $M$ different social networks. The networks are static throughout the simulation. On each network an agent can be either active or inactive being that an agent can only be active in one network (context $C_{k}$ ) at a time.

On each simulation cycle, the $N$ agents are schedule to execute their behaviour sequentially and in a uniform random order. The behaviour of the current agent $i$, located in the context $C_{k}$ can be described as follows:

1. Choose an available neighbour (agent $j$ ) from the current context at random;

2. Update agent $i$ and $j$ opinions and uncertainties according to the equations 2 and 3 from the previous section 2.2;

3. Switch to a random distinct context $C_{l}\left(C_{l} \neq C_{k}\right)$ with a probability $\zeta_{C_{k}}$, which is a static parameter with different values for each context / network;

Note that although static complex social network models allow us to create abstract representation for social contexts can be relatively stable if we consider short to moderate periods of time, our social peers are not always available at all times and spend different amounts of time in distinct relations. 


\section{Experiment Design}

The simulation experiments were created using the MASON framework [16] and executed in a grid environment described in [17]. In each experiment, a population of 300 agents starts with random opinions with values drawn from a uniform distribution between -1 and 1 . They interact until 3000 cycles pass or the opinion values stabilise. We perform 30 simulation runs for each parameter combination. We have two main goals with these experiments. The first is to analyse the dynamics of opinion formation under the model of relative agreement described in the previous section. This model combines both the relative agreement interaction rules and the context switching social spaces with multiple contexts. The second goal is to analyse the influence of different network topologies in the formation of consensus in multi-agent societies.

In this paper, we present a set of experiments focused on the analysis of the dynamics induced by the context switching mechanism. We spanned the switching parameter $\left(\zeta_{C_{i}}\right)$ from 0 to 1 in intervals of 0.05 with two contexts. We also use different network topologies in these contexts. We then observe how different combinations of context switching probabilities and network structures affect the speed of convergence to stable opinion values.

The initial uncertainty parameter is set to $U=1.4$. According to the previous work with relative agreement in single social networks [1], this value guaranteed the convergence in one central opinion value. We chose this parameter value to ensure that interactions are not heavily restricted by the uncertainty early in a simulation run. We want to study the influence of different network topologies so these are the structures that intrinsically guide such interactions early on.

We tested our model with three network models: regular networks, with the same number of connections for each agent; scale-free network, generated using the Barabasi-Albert (BA) model [5]; small-world network, generated using the Watts \& Stroggatz (WS) model [23].

\section{Results and discussion}

In this section we present and discuss the experimental results. We show how different values of switching between contexts influence the speed of convergence to stable opinion values. We also explore the interplay between the model of relative agreement presented in section 2.3 and different network topologies.

\subsection{Context switching with regular networks}

In this set of experiments, we focus on the analysis of how the switching probabilities affect the opinion formation game. To do this, we construct several simulation scenarios where agents interact in two social relations. Each relation is associated with an abstract network model and has a context switching value $\zeta_{C_{i}}$. This value corresponds to the probability of switching from a relation to another (as described in section 2.3). 
We maintain homogeneous network structures and span the context switching values $\left(\zeta_{C_{i}}\right)$ from 0 to 1 in intervals of 0.05 . Figure 3 depicts a landscape for this parameter span. In this case we create two contexts each one with a $k$ regular network with $k=30$. Regular networks offer an easy way to model highly clustered populations of agents. For this type of networks each node is connected with $2 k$ other nodes, where $k$ is a parameter of the generative procedure. Regular networks also provide a convenient way to observe the influence of neighbourhood size in the opinion stabilisation process as the connectivity structure is equal for all the agents. They can also serve as models for highly clustered communities (although its structure is far from real world scenario topologies $[23,5]$ ).
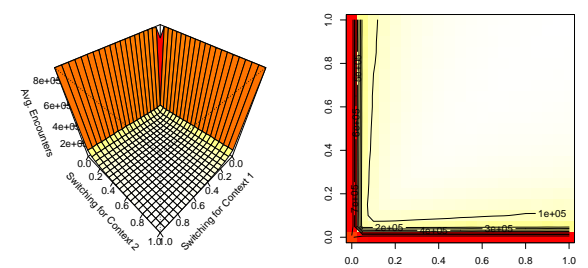

Fig. 3: Meetings to achieve convergence to stable opinion values with two 30-regular networks and $\zeta \in[0,1]$.

In figure 3 , we see that small probabilities $\left(0 \leq \zeta_{C_{i}} \leq 0.1\right.$ proximately) in one of the contexts lead to a large numbers of encounters necessary to stabilise the opinion values. In extreme cases, stabilisation is never reached. We can also observe the configuration $\left(\zeta_{C_{1}}, \zeta_{C_{2}}\right)=(0,0)$ is slightly better in these cases. This is because agents are isolated in each context and thus the opinions evolve separately the same way they would if the agents were placed within a single network. Also note that although not depicted in the figure, in this last case the opinions also evolve to two separate values.

Figure 4a shows a zoom in the previous landscape (figure 3 ) with the $\zeta$ being between 0.2 and 1 . This is the optimal zone in terms of encounters necessary to achieve stable opinions. Here we can see that the optimal values for switching with this regular configuration lies within $\zeta_{C_{i}} \in[0.8,1]$ proximately. Moreover, if one of the contexts has a high switching probability, the other context should have a similar level of switching.

Having one social relation with high switching while having the second with a low probability leads to a scenario where agent spend most of the time in one context but can still switch to another one. While they spend considerately less simulation time in this second context, this is enough to destabilise the opinion formation process.

In the next experiment we created a scenario to observe the effects of different connectivity levels for each context. Figure $4 \mathrm{~b}$ depicts the span of the switching probability within the values $\zeta \in[0.2,1]$. The first context is now a 10-regular 

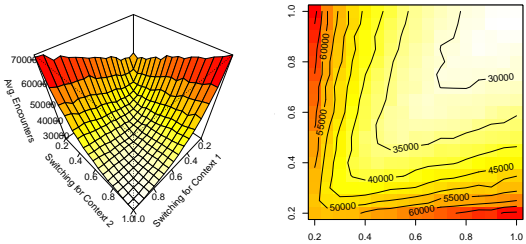

(a) networks: 30-regular / 30-regular
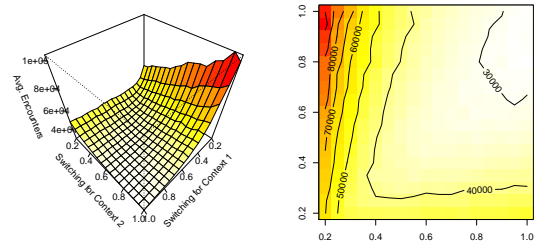

(b) networks: 10-regular / 50-regular .

Fig. 4: Meetings to achieve convergence to stable opinion values with for $\zeta \in[0.2,1]$. In the first landscape (figure 4a) we use 30-regular networks in both contexts. In figure $4 \mathrm{~b}$ we use a 10-regular and a 50-regular for context 1 and 2 respectively.

network (each agent has 20 neighbours) while the second is a 50-regular network (each agent having a total of 100 neighbours).

As we can see the asymmetry in the connectivity has clear effects in the convergence to stable opinion values. In this case, we find that if an agent stays more time $(\zeta \in[0.2,0.3])$ in the context with the lowest connectivity it seems to be important to switch less frequently from the highly connected and clustered social layer. Similarly to what was found in [17], a possible explanation is that in larger neighbourhoods, the probability of performing encounters with an agent with a very different opinion early in the simulation is considerable. The impact is clearly visible as disturbance in the convergence to stable opinions.

\subsection{It's a small world after all}

One evidence of the importance of network structure can be found in the next results. We conducted experiments using the Watts \& Strogatz (WS) model [23] to generate networks with small-world properties. These topologies are constructed by rewiring regular networks, introducing increasing amounts of disorder. Moreover, we can construct highly clustered networks, like regular lattices, yet with small characteristic path lengths, like random graphs. They are called smallworld by analogy with the phenomenon [22], popularly known as six degrees of separation. This phenomena refers to the idea that everyone is on average approximately six steps away, by way of introduction, from any other person on Earth.

Figure 5 shows the results for a set-up with two WS networks with an initial $k=30$ and a rewiring probability of $p=0.1$ and $p=0.6$. The value of $p=0.1$ for the rewiring, introduces enough disorder in the network to lower the average path length without sacrificing the clustering coefficient too much. In figure 5a, we can see that the influence is very similar to the previous results with regular networks (see figure 4a) but the reduction in the path length causes the model to converge more rapidly for higher switching probabilities. 


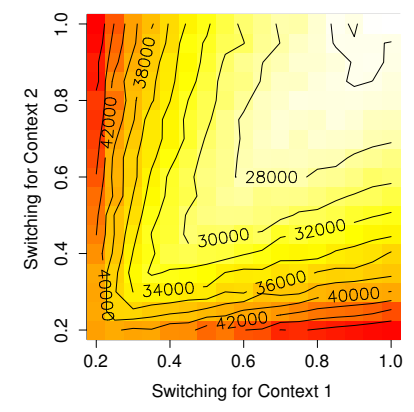

(a) WS network with $p=0.1$.

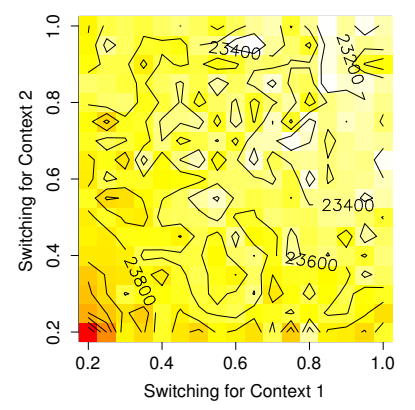

(b) WS network with $p=0.6$

Fig. 5: Meetings to achieve convergence to stable opinion values for two Watts \& Strogatz small-world networks generated with initial degree $k=30$ and rewiring probability $p=0.1$ (5a) and $p=0.6$ (5a). The switching values are $\zeta \in[0.2,1]$.

When we increase the level of disorder, for instance, to a value of $p=0.6$, the network clustering coefficient is significantly reduced, while maintaining the low average path length. The results for this are depicted in figure 5b. Although the switching probability seems to have a more complex influence on the speed of convergence, globally, the number of encounters seem to be almost homogeneous throughout the switching vales $\zeta_{C_{i}}>0.3$. Also, as the number of necessary encounters is a slightly lower, it seems that high values of switching are more important when the networks possess highly clustered nodes.

\subsection{Context switching with scale-free networks}

In this section we briefly discuss the results for the experiments with the scalefree network models. We performed an experiment with two contexts each one with a scale-free network. In this network, each node has a minimum connectivity of 1 , meaning that the preferential attachment mechanism only actuates once each time a node is added to the network. This thus generates a network with a forest topology.

Figure 6 shows that although the majority of nodes has a very low connectivity (see, [5]), the small-world characteristics of this scale-free model provide means to achieve convergence to stable opinion values. This happens for switching probabilities approximately within $\zeta_{C_{i}} \geq 0.1$, much like what happens in the previously described experiments. 

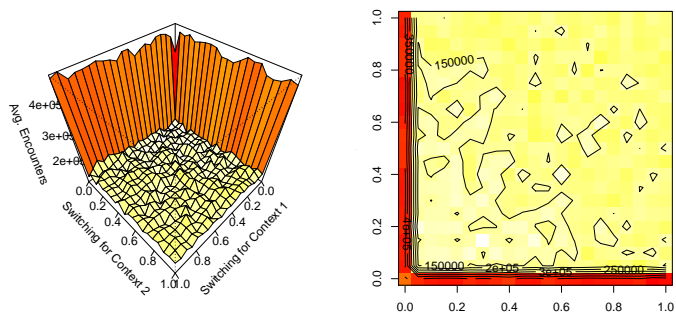

Fig. 6: Meetings to achieve convergence to stable opinion values for two scale-free networks with minimum degree $k=1$. The switching values are $\zeta \in[0,1]$.

\subsection{Results with a single network}

For result comparison purposes we performed a series of experiments with single networks. Using a parameter $k$ with the values $k=\{1,2,3,4,5,10,20,30,40,50\}$, we performed experiments with single k-regular, WS small-world and BA scalefree networks (see figure 7). For the regular networks the parameter $k$ is the previously described connectivity with each agent having $2 k$ neighbours. For the small-world networks, this parameter is used to construct the initial k-regular structure, we used a rewiring probability $p=0.1$ to keep these network highly clustered. For the BA scale-free networks the $k$ is the minimum degree each agent will have upon generating the network instance.

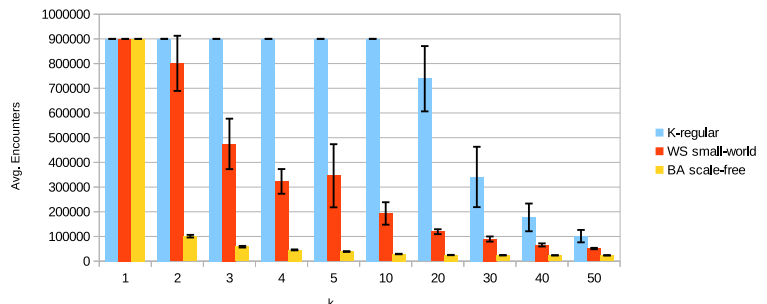

Fig. 7: Meetings to achieve convergence to stable opinion values with a single network context (without switching). Results for k-regular, WS small-world with $p=0.1$ and BA scale-free networks.

Note that in figure 7 , the maximum value of encounters is limited by the maximum number of simulation cycles allowed. In this case, the models that display the maximum number of encounters did not converge to stable opinion values. 
In figure 7 we can see that for $k \geq 2$, scale-free networks seem to outperform the other models in terms of convergence speed. Also note that these results confirm that the switching mechanism allows the opinion formation process to converge both faster and more often. As an example, consider the results for scale-free networks (figure 6) where convergence was made possible by exposing the agents to two distinct contexts.

The results in this paper show that the usage of different network model structures plays an important role when modelling opinion or consensus formation processes. Context dynamics seems to be an advancement as a modelling methodology for complex real-world scenarios and has a deep influence in how simulation models behave. These are key points discussed both in the work of Antunes et al. $[3,17]$ and Amblard et al. $[6,1]$ from which this work stems from.

\section{Conclusion and future work}

The results in this paper corroborate the fact that multiple context structures play an important role in processes such as opinion formation. While complex network models are good for modelling real-world social relation scenarios, single network structures fail to capture the complexity of the multitude of existing relations. Social decision making and the phenomena associated with this processes are influenced in different ways by distinct kinds of social relations. Examples of this are found in real-world events such as contemporary political or marketing campaigns.

The model here presented, while abstract by nature, can unveil interesting dynamics that should be taken into account when modelling complex social spaces for simulation models. The switching probability also introduces a way to model interaction temporal dynamics by allowing the modelling of time agents dedicate to different social contexts and how this affects the formation of opinions.

For future work, we will extend the presented exploration to include heterogeneous context configurations, combining different social network models. We also consider to explore scenarios where the uncertainty is heterogeneous [1].

\section{References}

1. Frédéric Amblard and G. Deffuant. The role of network topology on extremism propagation with the relative agreement opinion dynamics. Physica A: Statistical Mechanics and its Applications, January 2004.

2. Luis Antunes, João Balsa, Paulo Urbano, and Helder Coelho. Exploring context permeability in multiple social networks. In Keiki Takadama, Claudio CioffiRevilla, and Guillaume Deffuant, editors, Simulating Interacting Agents and Social Phenomena, volume 7 of Agent-Based Social Systems, pages 77-87. Springer Japan, 2010.

3. Luis Antunes, Davide Nunes, Helder Coelho, João Balsa, and Paulo Urbano. Context Switching versus Context Permeability in Multiple Social Networks. In Progress in Artificial Intelligence, volume 5816 of Lecture Notes in Computer Science, pages 547-559, 2009. 
4. Robert Axelrod. The Dissemination of Culture. Journal of Conflict Resolution, 41(2):203-226, 1997.

5. A.L. Barabási and R. Albert. Emergence of scaling in random networks. science, 286(5439):509, 1999.

6. Guillaume Deffuant, Frédéric Amblard, Gérard Weisbuch, and Thierry Faure. How can extremism prevail? A study based on the relative agreement interaction model. Journal of Artificial Societies and Social Simulation, 5(4):1, 2002.

7. Guillaume Deffuant, David Neau, Frederic Amblard, and Gérard Weisbuch. Mixing beliefs among interacting agents. Advances in Complex Systems, 3(01n04):87-98, 2000 .

8. Morris H. Degroot. Reaching a Consensus. Journal of the American Statistical Association, 69(345):118-121, 1974.

9. Naomi Ellemers, Russell Spears, and Bertjan Doosje. Self and social identity*. Annual Review of Psychology, 53(1):161-186, 2002.

10. J.P.R. Jr. French. A formal theory of social power. Psychological Review, 63:181194, 1956.

11. Serge Galam. Rational group decision making: A random field ising model $\mathrm{t}=0$. Physica A: Statistical Mechanics and its Applications, 238(1):66-80, 1997.

12. Rainer Hegselmann and Ulrich Krause. Opinion dynamics and bounded confidence: models, analysis and simulation. J. Artificial Societies and Social Simulation, $5(3): 2,2002$.

13. Wander Jager and Frédéric Amblard. Uniformity, bipolarization and pluriformity captured as generic stylized behavior with an agent-based simulation model of attitude change. Computational \& Mathematical Organization Theory, 10:295303, 2004.

14. Ulrich Krause. A Discrete Nonlinear and Non-Autonomous Model of Consensus Formation. In Communications in Difference Equations, pages 227-236. Gordon and Breach Pub., Amsterdam, 2000.

15. Keith Lehrer. Social consensus and rational agnoiology. Synthese, 31:141-160, 1975.

16. Sean Luke, Claudio Cioffi-Revilla, Liviu Panait, Keith Sullivan, and Gabriel Balan. MASON: A Multiagent Simulation Environment. Simulation, 81(7):517-527, 2005.

17. Davide Nunes and Luis Antunes. Consensus by segregation - the formation of local consensus within context switching dynamics. Proceedings of the 4 th World Congress on Social Simulation, WCSS 2012, 2012.

18. Sonia Roccas and Marilynn B. Brewer. Social identity complexity. Personality and Social Psychology Review, 6(2):88-106, 2002.

19. Thomas C. Schelling. Models of segregation. The American Economic Review, 59(2):pp. 488-493, 1969.

20. Thomas C. Schelling. Dynamic models of segregation. Journal of Mathematical Sociology, 1:143-186, May 1971.

21. Herbert A. Simon. Bandwagon and underdog effects and the possibility of election predictions. The Public Opinion Quarterly, 18(3):pp. 245-253, 1954.

22. Jeffrey Travers and Stanley Milgram. An experimental study of the small world problem. Sociometry, 32(4):pp. 425-443, 1969.

23. Duncan J Watts and Steven H Strogatz. Collective dynamics of 'small-world' networks. Nature, 393(6684):440-442, 1998.

24. G. Weisbuch. Bounded confidence and social networks. The European Physical Journal B-Condensed Matter and Complex Systems, 38(2):339-343, 2004.

25. Haoxiang Xia, Huili Wang, and Zhaoguo Xuan. Opinion dynamics: A multidisciplinary review and perspective on future research. IJKSS, 2(4):72-91, 2011. 